\title{
Práticas de enfermagem associadas às dinâmicas de prevenção ao suicídio: Um relato de experiência
}

\author{
Nursing practices associated with suicide prevention dynamics: An experience report \\ Prácticas de enfermeira asociadas a la dinâmica de prevención del suicidio: Un relato de \\ experiencia
}

Ana Karina Rodrigues Coelho ORCID: https://orcid.org/0000-0001-8504-689X Centro Universitário Fibra, Brasil

E-mail:anna.karinna1999@gmail.com

Amanda Guimarães Cunha ORCID: https://orcid.org/0000-0002-0893-3281

Centro Universitário Fibra, Brasil

E-mail: amandaguic18@gmail.com

Ana Beatriz Cravo da Silva

ORCID: https://orcid.org/0000-0002-3626-2518

Centro Universitário Fibra, Brasil

E-mail: beas1b@outlook.com

Andrey Santana Cid

ORCID: https://orcid.org/0000-0001-9655-4048

Centro Universitário Fibra, Brasil

E-mail: andreycid214@gmail.com

Luna Carolina Cardoso Castro

ORCID: https://orcid.org/0000-0002-2368-7867 Centro de Atenção Psicossocial Álcool e outras drogas, Brasil

E-mail: luacastro2014@gmail.com

Bárbara Cybelle Monteiro Lopes

ORCID: https://orcid.org/0000-0001-7122-2872

Universidade Federal do Pará, Brasil

E-mail: barbaracml95@gmail.com

Barbara Maria Neves Mendonça Luz

ORCID: https://orcid.org/0000-0001-7907-9337

Centro Universitário do Estado do Pará, Brasil

E-mail: barbaramendoncaluz@ outlook.com

João de Souza Mendonça

ORCID: https://orcid.org/0000-0002-2204-5010

Universidade da Amazônia, Brasil

E-mail: joaosm.farmaco@gmail.com

Larissa Pantoja Silva

ORCID: https://orcid.org/0000-0003-4424-5176

Centro Universitário do Estado do Pará, Brasil

E-mail: larissapantoja24@gmail.com

Leidiane de Jesus da Costa Santos

ORCID: https://orcid.org/0000-0002-9208-2319

Escola Superior da Amazônia, Brasil

E-mail: leidianesantos2023@gmail.com

Suely Patrícia Perdigão de Abreu

ORCID: https://orcid.org/0000-0001-9802-6943

Universidade da Amazônia, Brasil

E-mail: suelypatricia8@gmail.com

Tamires Costa Franco

ORCID: https://orcid.org/0000-0002-8978-4176

Universidade da Amazônia, Brasil

E-mail: tamiresfranco98@gmail.com

Tâmia Rayara Carvalho Araujo da Silva ORCID: https://orcid.org/0000-0003-4713-7314

Universidade da Amazônia, Brasil E-mail: ta.mia10@hotmail.com 


\begin{abstract}
Resumo
O comportamento suicida refere-se a uma serie de fenômenos relacionados ao suicídio, dos quais os mais relevantes são: o pensamento em se matar, denominada de ideação suicida, o suicídio propriamente dito (óbito) e a tentativa de suicídio, quando a morte não é efetivada. Portanto, compete aos profissionais de enfermagem aplicar condutas a partir do conhecimento científico em saúde mental, para impedir a efetivação de tal ato. Com esse estudo, objetiva-se relatar a experiência em um grupo de educação em saúde, a partir de atividade dinâmica alusiva ao setembro amarelo. Tratase de um estudo descritivo com abordagem qualitativa na modalidade relato de experiência, onde foi aplicada a observação participante de acadêmicos de enfermagem durante a realização do grupo de educação em saúde em um Centro de Atenção Psicossocial Álcool e Drogas (CAPS AD). Nas atividades dinâmicas, os usuários, receberam balões e a missão de manter o balão a "salvo", entretanto, foram motivados a movimentar o corpo e o balão. Em seguida, foram entregues perguntas com questionamentos, os quais eles precisavam resolver em vinte segundos, caso não conseguissem, deveriam estourar o balão. Posteriormente, os usuários foram orientados de que o balão representava suas vidas, gerando uma reflexão, sobre problemas, resoluções e suicídio, devendo cada um expressar no papel como se sentiam a partir da reflexão exposta. Ao longo da atividade dinâmica os usuários participaram de forma ativa, sendo orientados a pedir ou procurar ajuda quando apresentarem dificuldades de resolutividade das crises, e evitar o uso de Substâncias Psicoativas (SPA) como estratégias adaptativas para enfrentar o sofrimento.
\end{abstract}

Palavras-chave: Suicídio; Usuários dependentes; Assistência à saúde mental.

\begin{abstract}
Suicidal behavior refers to a series of suicide-related phenomena, the most relevant of which are suicide itself (death) and attempted suicide. Therefore, it is up to nursing professionals to apply behaviors based on scientific knowledge in mental health, to prevent the realization of such an act. With this study, the objective was to report the experience in a health education group based on the dynamic activity alluding to the yellow September. This is a descriptive study with a qualitative approach in the experience reporting modality, in which participant observation by nursing students during the health education group was applied. In dynamic activities, users received balloons and the mission to keep the balloon "safe", however, they were motivated to move the body and the balloon. Then, questions were asked with questions, which they needed to solve in twenty seconds, if they couldn't, they should pop the balloon. Subsequently, users were told that the balloon represented life, generating a reflection on problems, resolutions and suicide, each of whom must express on paper how they felt from the reflection exposed. Throughout the dynamic activity, users participated actively, being instructed to ask for or seek help when they present difficulties in resolving crises, and to avoid using psychoactive substances as adaptive strategies to face suffering.
\end{abstract}

Keywords: Suicide; Drug users; Mental health assistance.

\title{
Resumen
}

La conducta suicida se refiere a una serie de fenómenos relacionados con el suicidio, siendo los más relevantes el suicidio mismo (muerte) y el intento de suicidio. Por tanto, corresponde a los profesionales de enfermería aplicar comportamientos basados en el conocimiento científico en salud mental, para evitar la realización de tal acto. Con este estudio, el objetivo fue reportar la experiencia en un grupo de educación para la salud a partir de la dinámica de actividad alusiva al septiembre amarillo. Se trata de un estudio descriptivo con abordaje cualitativo en la modalidad de relato de experiencias, en el que se aplicó la observación participante por estudiantes de enfermería durante el grupo de educación en salud. En las actividades dinámicas, los usuarios recibieron globos y la misión de mantener el globo "seguro", sin embargo, se motivaron a mover el cuerpo y el globo. Luego, se hicieron preguntas con preguntas, que debían resolver en veinte segundos, si no podían, deberían hacer estallar el globo. Posteriormente, se informó a los usuarios que el globo representaba la vida, generando una reflexión sobre problemas, resoluciones y suicidios, cada uno de los cuales debe expresar en papel cómo se sintieron a partir de la reflexión expuesta. A lo largo de la actividad dinámica, los usuarios participaron activamente, instruyéndose para que pidan o busquen ayuda cuando presenten dificultades para resolver crisis, y eviten el uso de sustancias psicoactivas como estrategias adaptativas para afrontar el sufrimiento.

Palabras clave: Suicidio; Consumidores de drogas; Atención a la salud mental.

\section{Introdução}

O suicídio se constitui como um dos mais antigos temas relacionados à saúde dos indivíduos e a forma como são afetados pelas sociedades e coletividades nas quais vivem. Em termos históricos, sua relevância no plano social pode ser identificada desde a Grécia antiga. Em tempos modernos, ao menos desde o século XVIII, e tem sido tratado como fenômeno social (Ribeiro \& Moreira, 2018).

Segundo Bertolote, o suicídio pode ser definido como " ato deliberado" intencional, de causar morte a si mesmo; iniciado e executado por uma pessoa que tem clara noção ou forte expectativa de que o desfecho seja fatal e resulte em sua 
própria morte. O suicídio é compreendido atualmente como um fenômeno multidimensional, que resulta de uma interação complexa entre fatores ambientais, sociais, fisiológicos, genéticos e biológicos, sendo considerado um tema tabu em muitas sociedades (Cescon et al., 2018).

O sofrimento emocional é carregado de estigmas, com isso, as pessoas tem vergonha de admitir suas angustias e aflições; admitir e expressar o que se passa em seus pensamentos uma forte ideia de que a morte seria um alívio para o sofrimento, uma forma de saída dos conflitos que costuma ser escondida ou camuflada, dificultando ainda mais o acesso e ajuda, ou suporte especializado a esse indivíduo (Fontenelle, 2008).

As taxas de tentativas de suicídio são aproximadamente dez vezes maiores que as taxas de suicídios consumados. O suicídio não é um ato com mecanismos bem esclarecidos, envolve diversos fatores de risco que precisa de atenção e compreensão em um contexto social e comportamental (Abreu et al., 2010).

O tema suicídio demanda uma alta atenção dos profissionais de saúde, para que possam tratar dos riscos e das possibilidades de prevenção. Sendo preciso levar em consideração as situações sociais, como empregabilidade e desemprego, estrutura familiar, condições socioeconômicas, aceitando no meio de convivência, entre outros fatores (Abreu et al., 2010).

O suicídio se tornou um grave problema de saúde pública, pois hoje, cerca de $75 \%$ da população mundial apresenta sofrimento psíquico, ocorrendo 800 mil mortes a ano, sendo visualizado que para cada uma pessoa que efetiva o suicídio, muitas outras possuem a tentativa, havendo maior prevalência em pessoas de baixa e média renda. Atualmente, como principais instrumentos para tentativa de suicídio, OPAS (2020) destaca o uso de pesticidas, enforcamento e o uso de arma de fogo.

Os transtornos mentais mais comuns associados ao suicídio são: depressão, transtorno do humor bipolar e dependência de álcool e de SPA. A esquizofrenia e sofrimentos psíquicos relacionados à personalidade também são fatores de riscos importantes. Essa situação de risco se agrava quando uma dessa condições se associam à depressão e alcoolismo, ou ainda a coexistência de depressão, ansiedade e agitação (Bertolote \& Fleischmann, 2002).

Com esse estudo, objetiva-se relatar a experiência de acadêmicos de enfermagem em um grupo de educação em saúde, a partir da atividade dinâmica alusiva ao setembro amarelo, cuja proposta era a mitigação de dúvidas em relação a associação de álcool e SPA com o suicídio.

\section{Metodologia}

A construção do conhecimento científico organiza-se numa multiplicidade, permanentemente desafiada a elaborar modos de leituras sobre a realidade, envolvendo ideologias, metodologias, interações dialógicas entre sujeitos, contextos e pesquisadores, além de concepções sociopolíticas e históricas (Demo, 1995). A referente pesquisa trata-se de um estudo descritivo com abordagem qualitativa, na modalidade relato de experiência, a partir de uma atividade de educação em saúde com um grupo de usuários em um Centro de Atenção Psicossocial Álcool e outras Drogas (CAPS AD).

Tendo o conhecimento que o referido CAPS AD oferece atendimento por meio de uma equipe multiprofissional, que conta com enfermeiros, psiquiatras, psicólogos, assistentes sociais, terapeutas ocupacionais e nutricionistas, ofertando serviços que asseguram o tratamento e acompanhamento da saúde física e mental não somente aos usuários, como também as suas famílias, garantindo suporte através de grupos terapêuticos e de forma individual, respeitando suas individualidades e demandas.

A atividade vivenciada pelos discentes ocorreu durante as práticas de estágio curricular obrigatório do componente curricular da disciplina de Enfermagem em Saúde Mental, no qual evidencia-se a relevância da promoção e prevenção à saúde mental, bem como aspectos que envolvem a Sistematização da Assistência de Enfermagem (SAE), os quais efetivam os cuidados necessários visando a individualidade de cada usuário, ao se ofertar um cuidado equânime, integral e acessível. A 
ação decorreu no mês de setembro, a partir da liberação da gerência do local e com a supervisão de uma das enfermeiras do CAPS AD.

Diante do contexto do mês de setembro ser em alusão a campanha sobre a prevenção e conscientização do suicídio juntamente com a experiência da prática, percebeu-se a oportunidade da realização de uma dinâmica, voltada tanto para a conscientização sobre o suicídio quanto para explanar a importância da vida.

A idealização do setembro amarelo se iniciou a partir do surgimento da história de Mike Emme, nos Estados Unidos. Em 1994 o rapaz, de apenas dezessete anos cometeu suicídio, não havendo, pela família ou amigos, identificação ou reconhecimento de sinais que poderiam sugerir que o mesmo iria cometer o ato. A partir deste momento, a divulgação de que se faz necessária ajuda em casos de tentativas de suicídio, passou a ser disseminada pelo mundo todo, apresentando como símbolo, o laço amarelo, adorno usado nas cestas decoradas no enterro de Mike, representando a cor do carro do mesmo. Em 2003, a Organização Mundial da Saúde (OMS), instituiu o dia 10 de setembro como o dia de prevenção e conscientização sobre este ato que assola pessoas no mundo todo e que vem se tornando uma das principais causas de morte atualmente (OPAS, 2020).

Dentre as fases ocorridas até a concretização da atividade de educação em saúde, houve inicialmente uma conversa com a enfermeira e preceptora em consenso com os discentes para que juntos decidissem a atividade que deveria ser realizada, além de uma maneira de atrair os usuários para a atividade, bem como fazer uso de um modelo alternativo de ensino, como dinâmica ativa. Dito isso, sucedeu-se o convite aos usuários presentes no respectivo dia da ação, bem como se estendeu o mesmo aos acompanhantes que se faziam presentes.

A ação dividiu-se em dois momentos específicos, em um mesmo local, ao qual foi cedido pelo próprio centro: no primeiro houve uma explanação sobre a história do setembro amarelo tal qual sua influência nos dias atuais, e no segundo realizou-se uma dinâmica com a utilização de balões, música, quiz sobre diferentes curiosidades, lápis de cor e folhas A4. Ao todo compareceram cerca de 10 usuários frequentadores do CAPS AD, 2 acompanhantes, os respectivos discentes e a enfermeira responsável.

\section{Resultados}

Durante o mês de setembro, mês de alusão ao setembro amarelo, foi realizado no CAPS AD, uma roda de conversa, na qual pode-se notar a particularidade de cada usuário ao relatar seus pensamentos, seja por conta do álcool ou outras SPA, problemas familiares e/ou sociais, entre outros.

Foi elaborado uma atividade dinâmica devido ao número de casos de suicídio sem definição específica de idade, pois notou-se que muitos usuários em suas consultas de enfermagem relatavam queixas de convívio social apenas. Na atividade dinâmica esses usuários receberam balões e a missão era manter o balão a "salvo", não informando os usuários sobre o real significado do balão na dinâmica. Foram realizados três pontos significativos na dinâmica, pontos estes que trouxeram reflexão sobre a vida.

O primeiro ponto se fez presente ao serem entregues os balões e ao som da música eles foram orientados a se movimentar no ritmo da música sem deixar os balões caírem. O segundo momento reflexivo ocorreu a partir da entrega de perguntas com questionamentos, com a seguinte regra: quem não soubesse responder à pergunta deveria estourar o balão. Cada pergunta era diferente para cada usuário, sendo perguntas consideráveis fáceis, como por exemplo: "quem descobriu o Brasil?", "Quantos grãos de areia têm no deserto?"' e quem não soubesse responder, teria que estourar o balão.

Foram realizadas as perguntas, e algumas pessoas não souberam responder, e estouraram o balão. Após a conclusão de todos com suas respostas ou não, foi informado que o balão significava a vida de cada um deles. O terceiro ponto reflexivo da dinâmica, englobou uma roda de conversa, onde os usuários relataram sobre suas angústias, sobre o motivo de estarem em 
tratamento em um CAPS AD, e alguns que se sentiram confortáveis em compartilhar suas histórias, relatando que há poucos dias antes da atividade, tentaram realizar o suicídio e não tiveram apoio de nenhum familiar para evitar isso.

Pode-se notar que entenderam de forma positiva o que foi repassado, trocando ideias e relatando seus casos, já que muitos ali estavam em tratamento para fazer um desfecho de largar álcool e outras substâncias psicoativas. A importância de refletir sobre o suicídio nesse momento tão importante é fundamental, já que na maioria das vezes esse assunto é silenciado pela sociedade, e principalmente por familiares, o que pode se tornar um grave problema na vida de quem possui algum tipo de sofrimento psíquico, como a depressão ou de quem apresenta ideação suicida.

Ao prestar atendimento a esses usuários, se faz necessário haver um suporte emocional, qualidade e segurança no serviço, para que esses usuários aceitem melhor o atendimento e possam estar compartilhando o que lhes causa angústia. Desta maneira, o momento de acolhimento e de esclarecimentos de dúvidas com relação ao suicídio e algumas de suas características que podem ser notadas, sendo possível identificar na fisionomia de cada um que se encontrava no encontro, a importância de poder ter uma escuta qualificada, apoio emocional e assistência integral.

\section{Discussão}

O suicídio é mais do que um desfecho de um comportamento pessoal, passando a ser um problema sociocultural e psicossocial. Devido as suas características multifacetadas, o suicídio é um evento que deve ser enfrentado por meio de iniciativas interdisciplinares e intersetoriais (Fontão et al., 2018).

A falta de informação e o preconceito com quem sofre de depressão, ansiedade ou outros sofrimentos psíquicos pode resultar no suicídio da pessoa. É importante que todos os profissionais sejam qualificados e atuem de forma humanizada para atender a particularidade de cada indivíduo, a fim de proporcionar conforto e segurança para que possam falar o que está lhe angustiando.

Com base na experiência vivida de proporcionar uma roda de conversa em relação à importância da vida para esses usuários que no decorrer da conversa se mostraram tristes e angustiados por situações pessoais e sociais, pode-se perceber a importância da escuta qualificada e aberta, sem julgamentos, como estratégia terapêutica e de resolubilidade de crises situacionais, com a construção de insights.

Os profissionais de saúde devem acolher e identificar, o quanto antes, esses riscos de suicídio, para que haja prevenção e tratamento, além de inclui-los em programas de acompanhamento para que preservem a vida. Com o aumento de suicídio nos últimos anos, o papel da enfermagem na primeira linha de cuidado se tornou mais que importante, e se caracterizou com uma peça fundamental para ajudar prevenir os casos de suicídio.

$\mathrm{Na}$ atual conjuntura, o modelo de ensino tradicional vem sendo gradativamente substituído por novas tendências e implicações as quais apontam para a necessidade da formação de um profissional crítico-reflexivo, capaz de transverter a realidade social do seu cotidiano em benefícios acessíveis para a comunidade ao qual é inserido, tendo em vista proveitos coletivos que influenciarão diretamente na qualidade assistencial futura (Cunha et al., 2021).

Uma das principais dificuldades está relacionado à falta de confiança do paciente com o profissional, ou seja, o prejuízo do relacionamento interpessoal, já que muitas vezes, os julgamentos, o estigma sobre o tema, atuam como precursores de barreiras entre usuário e profissional de saúde mental.

Dessa forma, cabe ao profissional a responsabilidade de se fazer mais humano e com maior compreensão acerca de cada caso de suicídio, compreendendo suas especificidades e individualidade, identificando os desafios vivenciados pelos usuários, para que se possa obter um número de pessoas que consigam ressignificar suas angústias, sofrimentos e medos.

É importante lembrar que o suicídio é uma decisão pessoal de cada pessoa, caracterizado por ser um ato de "coragem" ou como um pedido de ajuda diante ao sofrimento interno que estão passando. Por somatizarem problemas, essas 
pessoas começam a desenvolver sofrimentos psíquicos que alteram sua percepção de realidade, a qual é visualizada como sem solução para os problemas, crises experimentadas. Na maioria das vezes essas pessoas com pensamento suicida procuram atendimento e até mesmo alguém próximo para desabafarem o que estão sentido naquele momento, e não são levadas a sério, o que é muito prejudicial.

Vale lembrar que se deve sempre manter atenção aos sinais de suicídio ou ideação suicida que uma pessoa pode apresentar, como: pensamentos negativos, alterações de humor, sentimento de culpa, desapego às atividades que gostava de fazer, entre outros, como o sentimento de desesperança.

\section{Conclusão}

Com base na experiência vivenciada e a partir dos relatos, evidenciou-se a necessidade do uso de atividades dinâmicas dentro dos serviços de saúde, tendo em vista o incentivo à participação ativa dos usuários, os quais contribuíram significativamente para que a dinâmica obtivesse o resultado esperado. Portanto, é importante frisar que os programas do CAPS e/ou outras instituições de apoio, ao promoverem rodas de conversar, campanhas de prevenção ao suicídio, e ao intensificarem a escuta qualificada, contribuem para a construção de uma rede de apoio acessível.

Ademais, legitimou-se neste estudo que o uso de atividades fora do consultório, principalmente as que se utilizam de dinâmicas e atividades alternativas são efetivas, já que proporcionam locais de escuta e fala dos usuários, promovendo sua autonomia em conjunto com outros que passam por situações semelhantes.

Para mais, é importante ratificar neste trabalho que os profissionais da enfermagem atuam na prevenção, promoção e tratamento tanto dos paciente como dos familiares, já que ao falar sobre saúde mental e suicídio, ambos são orientados a pedir ou procurar ajuda quando apresentarem dificuldades de resolubilidade de crises, e evitar o uso de substâncias psicoativas como estratégias adaptativas para enfrentar o sofrimento, como também são instruídos a reconhecer sinais indicadores, como inclinações suicidas dentro das famílias, já que ainda é considerado um tabu familiar e social.

Sugere-se para trabalhos futuros, que ocorram com um maior quantitativo de participantes, visando maior pluralidade de resultados e possibilitando assim, que este tipo de atuação seja abordada continuamente dentro dos Centro de atenção psicossociais, visando a atenuação no número de casos de suicídios efetivados, bem como, no que tange a atuação da enfermagem nos casos de tentativas de suicídio.

\section{Referências}

Abreu, K. P. d., Lima, M. A. D. S., Kohlrausch, E., \& Soares, J. F. (2010). Comportamento suicida: fatores de risco e intervenções preventivas. Rev. Eletr. Enf, 12(1), 195-200.

Barbosa, F. d. O., Macedo, P. C. M., \& Silveira, R. M. C. d. (2011). Depressão e o Suicídio. Rev. SBPH, 14(1), $233-243$.

Bertolote, J. M., \& Fleischmann, A. (2002). Suicide and psychiatric diagnosis: A worldwide perspective. World Psychiatry, 1, 181-185.

Bertolote, J. M. O suicídio e sua prevenção. Unesp, 2012.

Botega, N. J. (2014). Comportamento suicida: epidemiologia. Psicologia USP, 25(3), 231-236.

Cescon, L. F., Capozzolo, A. A., \& Lima, L. C. (2018). Aproximações e distanciamentos ao suicídio: analisadores de um serviço de atenção psicossocial. Saúde e Sociedade, 27(1), 185-200.

Cunha, A. G., Silva, A. F. L. d., Mendes, A. P. d. S., Oliveira, A. K. C. d., Braga, B. S. C., Silva, B. C. M. d., Portilho, D. C., Portilho, D. C., Nascimento, J. L. M., Silva, J. M. L. d., Soeiro, J. d. S., Ferreira, L. F., Carvalho, M. A., Ferreira, P. d. S., Carvalho, P. H. C. d., \& Silva, R. R. d. (2021). Uso de metodologias ativas na promoção do autocuidado e adesão terapêutica com usuários de um centro de atenção psicossocial. Research, Society and Development, 10(1), 1-7.

Demo, P. (1995). Metodologia Científica em Ciências Sociais (3a ed.). Editora Atlas.

Figueiro, M. \& Dimenstein, M. (2020). Rede de saúde mental e acolhimento para as famílias que sofrem com problemas decorrentes do uso de drogas no município do Natal, Rio Grande do Norte. Psicología, Conocimiento y Sociedad, 10(1), 81-100. 
Research, Society and Development, v. 10, n. 4, e50310413819, 2021

(CC BY 4.0) | ISSN 2525-3409 | DOI: http://dx.doi.org/10.33448/rsd-v10i4.13819

Fontão, M. C., Rodrigues, J., Lino, M. M., Lino, M. M., \& Kempfer, S. S. (2018). Cuidado de enfermagem às pessoas atendidas na emergência por tentativa de suicídio. Rev. Bras. Enferm, 71(5), 2329-35.

Fernandes, M. A., Silva, J. S. e., Campos, L. R. B., Nepomuceno, V. M. S., Vasconcelos, A. C. B., \& Oliveira, A. L. C. B. d. (2020). Prevenção ao suicídio: vivências de estudantes universitários. Revista Cuidarte, 11(2), 1-12.

Ribeiro, J. M., \& Moreira, M. R. (2018). Uma abordagem sobre o suicídio de adolescentes e jovens no Brasil. Ciênc. saúde coletiva, 23(9), 2821 -2834.

Santos, W. S. d., Ulisses, S. M., Costa, T. M. d., Farias, M. G., \& Moura, D. P. F. d. (2016). A influência de fatores de risco e proteção frente à ideação suicida. Psicologia, saúde \& doenças, 10(1), 515-526.

OPAS. (2020). Suicídio. Organização Pan Americana De Saúde. https://www.paho.org/pt/topicos/suicidio

OPAS. (2020). Pandemia de COVID-19 aumenta fatores de risco para o Suicídio. https://www.paho.org/pt/noticias/10-9-2020-pandemia-covid-19-aumentafatores-risco-para-suicidio. Acesso em: 29 de novembro de 2020. 